\title{
INÍCIO DA VIDA SEXUAL DE ADOLESCENTES DA ILHA DE SANTIAGO, CABO VERDE - ÁFRICA OCIDENTAL
}

\author{
BEGINNING OF SEXUAL LIFE OF ADOLESCENTS IN \\ SANTIAGO ISLAND, CAPE VERDE, WEST AFRICA
}

\author{
Carlos Mendes Tavares ${ }^{1}$, Paula Yuri Sugishita Kanikadan², \\ Airlane Pereira Alencar ${ }^{3}$, Néia Schor ${ }^{4}$
}

\begin{abstract}
RESUMO
Objetivo: estimar a idade de início da atividade sexual de adolescentes e sua associação com fatores sócio-demográficos. Método: estudo transversal, realizado entre janeiro e março de 2007, com amostra de 368 adolescentes de 13 a 17 anos de idade, sexualmente ativos, provenientes de oito escolas secundárias públicas da Ilha de Santiago, Cabo Verde, escolhidas aleatoriamente. $\mathrm{Na}$ análise das variáveis, foi ajustado um modelo de regressão múltipla para variável ordinal com função de ligação probit, considerando-se nível de significância de 5\%. Resultados: entre os 368 adolescentes, 31,5\% (116) eram meninas e $68,5 \%$ (252) meninos. Houve maior freqüência de iniciação sexual protegida entre os jovens que se iniciaram sexualmente com idade mais avançada (16 e 17 anos). A iniciação feminina foi mais tardia do que a masculina e rapazes mais novos (13 anos) relataram o início da vida sexual a partir de dez anos de idade. Após análise múltipla, quatro fatores permaneceram significativamente associados à idade do início da vida sexual no sexo feminino: idade, morar em casa alugada, não estar namorando no período da entrevista e menarca. Para o masculino foram: idade, não estar namorando no período da entrevista e interação entre idade e não namorar. Conclusões: a maior freqüência de iniciação sexual protegida ocorre entre os jovens que se iniciaram sexualmente com idade mais avançada. Todavia, a influência de morar em casa própria ou cedida e de parceria afetivo-sexual no início da vida sexual revela que a necessidade de ações de prevenção sejam direcionadas para segmentos com piores condições sócio-econômicas e para adolescentes que não namoram.
\end{abstract}

Palavras-chave: idade no início da vida sexual; saúde sexual e reprodutiva; Cabo Verde; África Ocidental.

1 Pesquisador em nível de Pós-doutorado da Faculdade de Saúde Pública da Universidade de São Paulo e atualmente, Professor Visitante da Carreira do Magistério Superior, Classe Adjunto, Junto ao Centro de Ciências da Saúde e do Desporto, Universidade Federal do Acre. E-mail: carlostavares@usp.br.

2 Farmacêutica. Doutorado na Faculdade de Saúde Pública, Universidade de São Paulo. E-mail: paulak@usp.br E-mail: paulak@usp.br.

3 Professora Doutora. Universidade de São Paulo - Instituto de Matemática e Estatística. E-mail: lane.alencar@gmail.com.

4 Professora Titular. Departamento de Saúde maTerno-infantil da Faculdade de Saúde Pública da Universidade de São Paulo. E-mail: neschor@usp.br.

Correspondência para: carlostavares@usp.br

Como citar este artigo: Tavares CM et al. Beginning of sexual life of adolescents in Santiago Island, Cape Verde, West Africa. Journal of Human Growth and Development 2011; 21(3): 771-779.

Artigo submetido em 08.01.11, aceito em 19.08.11. 


\begin{abstract}
Objective: to estimate the age of the first sexual intercourse and the effects of socio-demographic factors among adolescents. Method: cross-sectional study with 368 sexually active adolescents aged 13-17 years from eight public elementary and high schools, randomly selected, in Santiago Island, Cape Verde, in Jan-Mar/2007. The analysis was made by means of regression adjusted for ordinal variables with probit link function, with a $5 \%$ significance level. Results: among the 368 adolescents, 31.5\% (116) were female and $68.5 \%$ (252) were male. There was higher prevalence of protected sexual relationship among adolescents who began sexual life later (16 and 17 yrs). Sexual initiation among females occurred later and younger males (13 yrs) reported the first sexual intercourse from 10 years. After multiple analyses, four factors have remained statistically significant associated with the age of first sexual intercourse among females: age, living in rented house, not being dating and age at menarche. The factors included for males were age, not being dating and interaction between age and not being dating. Conclusions: adolescents who begin sexual life later have safe sexual relationships more frequently. However, the influence of living in their own or donated house and affective-sexual partnership at the beginning of sexual life reveals the necessity (or demand) of preventive actions. These actions must be focused on groups with worse socioeconomic conditions and adolescents who are not dating.
\end{abstract}

Key words: Age of first sexual intercourse; sexual and reproductive health; Cape Verde; West Africa.

\section{INTRODUÇÃO}

Com a iniciação das práticas sexuais que acompanham a maturação reprodutiva, os adolescentes estão mais sujeitos a gravidez e a infecções sexualmente transmissíveis (IST) devido a relações sexuais desprotegidas ${ }^{1}$.

Em Cabo Verde, entre 1990 e 2000, houve um aumento de $2,8 \%$ da taxa de fecundidade no grupo de adolescentes de 15 a 19 anos; em 1990, para cada 100 mulheres de 15 a 19 anos, 11,0 tiveram um filho, e em 2000, esse valor subiu para $11,3^{2}$.

$\mathrm{Na}$ maioria dos países subsaarianos, o uso de qualquer método contraceptivo não ultrapassa $20 \%$ entre adolescentes do sexo feminino casadas, embora mais de $60 \%$ declarem que não desejam ter filhos nos primeiros anos de casamento ${ }^{3}$.

$\mathrm{Na}$ adolescência ${ }^{4}$, período compreendido entre os 13 e 17 anos de idade, a maioria dos jovens tornam-se sexualmente ativos ${ }^{5}$. 0 início da vida sexual em idade muito jovem (antes dos 15 anos) tem sido mostrado como impor- tante fator de risco para gravidez na adolescência e aquisição de doenças sexualmente transmissíveis, incluindo o $\mathrm{HIV}^{6}$. A adolescência é a etapa da vida marcada por complexo processo de desenvolvimento biológico, psíquico e social. Nessa fase, as influências sociais externas à família tomam maior magnitude, pois vão implicar tomadas de decisões, de condutas e contribuir para a definição de estilos de vida ${ }^{5}$.

Em Cabo Verde, assim como em vários países do mundo, a primeira experiência sexual tem sido cada vez mais cedo, sendo mais cedo em adolescentes do sexo masculino ${ }^{3,7}$. Essa questão de gênero define também o tipo de vínculo com o primeiro parceiro e as decisões acerca das práticas contraceptivas, influenciando no comportamento de práticas mais ou menos seguras no relacionamento sexual ${ }^{8}$. Tais diferenças devem ser levadas em consideração na assistência e promoção da saúde reprodutiva para indivíduos nessa faixa etária.

A literatura sobre a sexualidade de adolescentes em países da África, sobretudo lusófonas, é escassa. No intuito de 
atualizar informações sobre a iniciação sexual de adolescentes de Cabo Verde, o objetivo é estimar a idade de início da atividade sexual de adolescentes e sua associação com fatores sociodemográficos.

\section{MÉTODO:}

O estudo faz parte de uma pesquisa transversal realizada com uma amostra probabilística e representativa de adolescentes residentes na Ilha de Santiago, em Cabo Verde, em 2007.

Cabo Verde é um arquipélago composto por dez ilhas (nove das quais habitadas) situado no oceano Atlântico a aproximadamente $500 \mathrm{~km}$ a oeste do continente africano, ocupando uma superfície de $4.033 \mathrm{~km}^{2}$. O estudo foi realizado na Ilha de Santiago, ilha com maior área de superfície terrestre $\left(991 \mathrm{~km}^{2}\right)$ e população, com cerca de 234.940 habitantes ${ }^{9}, 54 \%$ da população total.

Foi utilizada uma técnica de amostragem probabilística em dois estágios. No estágio 1 (amostragem por estratos) a estratificação foi feita por município, com sorteio de escolas por município. No estágio 2 (amostragem por conglomerado) a divisão foi por escolas, com sorteio de turmas do 70 ao $12^{\circ}$ ano de escolaridade (em todas as escolas selecionadas), resultando em listagem de todos os alunos com características de interesse, isto é, que obedecessem aos critérios de inclusão em cada classe sorteada. Ao todo, foram incluídas oito escolas públicas dentre as 16 existentes nos municípios da Ilha.

O tamanho amostral foi calculado baseando-se na percentagem de adolescentes sexualmente ativos $(83,6 \%)$, com erro amostral fixado em 3,0\% e com nível de confiança de 95,0\%. Partindo de uma base populacional de 25.618 escolares, o tamanho da amostra estimada foi de 576 adolescentes, à qual foram acrescidos 28,0\%, para compensar eventuais perdas. Assim, o tamanho da amostra final foi de 768 adolescentes de 13 a 17 anos, que frequentavam o nível secundário público, do $7^{\circ}$ ao $12^{\circ}$ ano de escolaridade, pois não havia registro de alunos dessa faixa etária matriculados em escolas privadas.

Em 2002, a Organização Mundial de Saúde ${ }^{4}$ definiu a adolescência como um recorte cronológico que encobre diferenças no processo de desenvolvimento, considerando adolescentes os indivíduos entre 10 e 19 anos de idade. Entretanto, tal definição necessita também abranger aspectos biológicos, psicológicos e econômicos vivenciados neste período do desenvolvimento ${ }^{10}$.

Todavia, foram considerados aptos à pesquisa apenas os adolescentes na faixa etária dos 13 aos 17 anos pelos seguintes motivos: algumas questões do instrumento estavam relacionadas às práticas sexuais; a faixa considerada representava o início e o processo médio da vida sexual de adolescentes; estava dentro da definição cronológica de adolescente adotada pelo Comitê de Peritos da $\mathrm{OMS}^{4}$ e também pelo fato de ser a faixa que concentrava mais da metade (58\%) dos escolares de nível secundário em todo o arquipélago. Além deste recorte, foram considerados apenas os jovens que já haviam iniciado sua vida sexual, resultando na exclusão de 400 adolescentes. Para este estudo foram considerados apenas os escolares sexualmente ativos (368).

Desse modo, os critérios de inclusão foram: ter idade entre 13 e 17 anos, estar regularmente matriculado e frequentar a escola secundária pública, ter iniciado a vida sexual e aceitar participar da pesquisa.

Utilizou-se um questionário autoaplicado, com 76 perguntas fechadas sobre dados sociodemográficos e comportamentais. Todos os adolescentes responderam ao questionário em sala de aula, com duração média de 50 minutos.

Após a coleta, o banco de dados foi construído no software CSPro 6.0, com dupla digitação. Na fase de análise da pesquisa, este foi convertido para o software Eviews 6.

A análise descritiva foi apresentada por meio de proporções, médias e desvio-padrão. A variável resposta foi a idade de início da primeira relação se- 
xual. As variáveis independentes foram fatores sociodemográficos como idade, tipo de casa, religião, tipo de fonte de abastecimento da água, já namorou antes do período da pesquisa, exposição à mídia (ter TV, rádio), idade da menarca, município de procedência e interação entre idade e não namorou. Para estudar a relação entre a variável resposta e as variáveis independentes foi ajustado um modelo de regressão múltipla para variável ordinal com função de ligação probit $^{11}$. Tal modelo leva em conta que a variável resposta é uma variável categórica, já que a idade de início da vida sexual pode assumir somente os valores, $12,13,14,15,16$ ou 17 anos.

O modelo probit para a variável observada Idade de Início da Vida Sexual (IVS) foi definido em função da variável (latente) não observada IVS* para a qual foi construído o modelo de regressão múltipla para variável ordinal:

$$
I V S^{*}=x^{\top} \beta+e,
$$

com as variáveis explicativas na matrix (x) e com e (erro aleatório) com distribuição gaussiana de média zero e homocedástico. foi tal que:

A variável resposta observada IVS

$$
\begin{aligned}
& \text { IVS }=12 \text {, se IVS } * \leq \gamma_{1} \\
& 13 \text {, se IVS* } \leq \gamma_{2} \\
& 14 \text {, se IVS* } \leq \gamma_{3} \\
& 15 \text {, se IVS* } \leq \gamma_{4} \\
& 16 \text {, se IVS* } \leq \gamma_{5} \\
& 17 \text {, se IVS* }>\gamma_{5}
\end{aligned}
$$

Os parâmetros do modelo $\beta$ e $\gamma$ e a variância de erro aleatório foram estimados pelo método de máxima verossimiIhança e o modelo foi ajustado no programa Eviews 6, conforme parametrização apresentada em Greene ${ }^{11}$.

Para interpretação dos coeficientes, quanto maior o coeficiente de uma determinada variável explicativa, maior a probabilidade da idade da vida sexual ser maior.

Foram poucos os relatos de adolescentes do sexo feminino que iniciaram a vida sexual antes dos 12 anos de idade, portanto foram reunidas em uma única categoria todas as jovens que iniciaram a vida sexual com 10 a 12 anos.

A pesquisa foi aprovada pelo Comitê de Ética em Pesquisa da Faculdade de Saúde Pública da Universidade de São Paulo e financiada pelo Programa de Estudantes-Convênio da Pós-Graduação PEC-PG/Capes.

\section{RESULTADOS}

A amostra de estudo foi constituída de 368 adolescentes. Entre eles, $31,5 \%$ (116) eram meninas e $68,5 \%$ (252) meninos. A média de idade do início da vida sexual para o sexo masculino foi $14(\mathrm{dp}=2,0)$ e $15(\mathrm{dp}=1,6)$ anos para o feminino. As percentagens de adolescentes que já haviam iniciado a vida sexual foram diferentes $(p<0,001)$ : $65,6 \%(252)$ e $30,2 \%$ (116), respectivamente.

A iniciação feminina foi mais tardia do que a masculina na amostra estudada; rapazes mais novos (com 13 anos) relataram o início da vida sexual desde os 10 ou 11 anos de idade.

As variáveis com $p>0,05$ na Tabela 1 foram retiradas do modelo reduzido de regressão múltipla para variável ordinal com função de ligação probit (Tabela 2). Após análise múltipla, quatro fatores permaneceram significativamente associados à idade do início da vida sexual para as adolescentes do sexo feminino: idade ( $p<0,001)$, morar em casa alugada $(p=0,001)$, não estar namorando no período da entrevista $(p=0,007)$ e idade da menarca $(p=0,004)$. Para os jovens do sexo masculino foram: idade $(p<0,001)$, não estavam namorando no período da entrevista ( $p=0,007)$ e interação entre idade e não namorar $(p=0,002)$.

Observou-se que a probabilidade de maior idade do início da vida sexual aumentou conforme a idade e esse efeito foi maior para adolescentes do sexo feminino (coeficiente $=0,72 ; \mathrm{EP}=0,14$ ) do que para os do masculino (coeficiente $=0,51 ; E P=0,06$ ). A idade foi uma variável que controlou o efeito de que 
Tabela 1: Estimativas do modelo completo regressão múltipla ordinal com função probit para idade do início da vida sexual, segundo sexo Ilha de Santiago, Cabo Verde, 2007

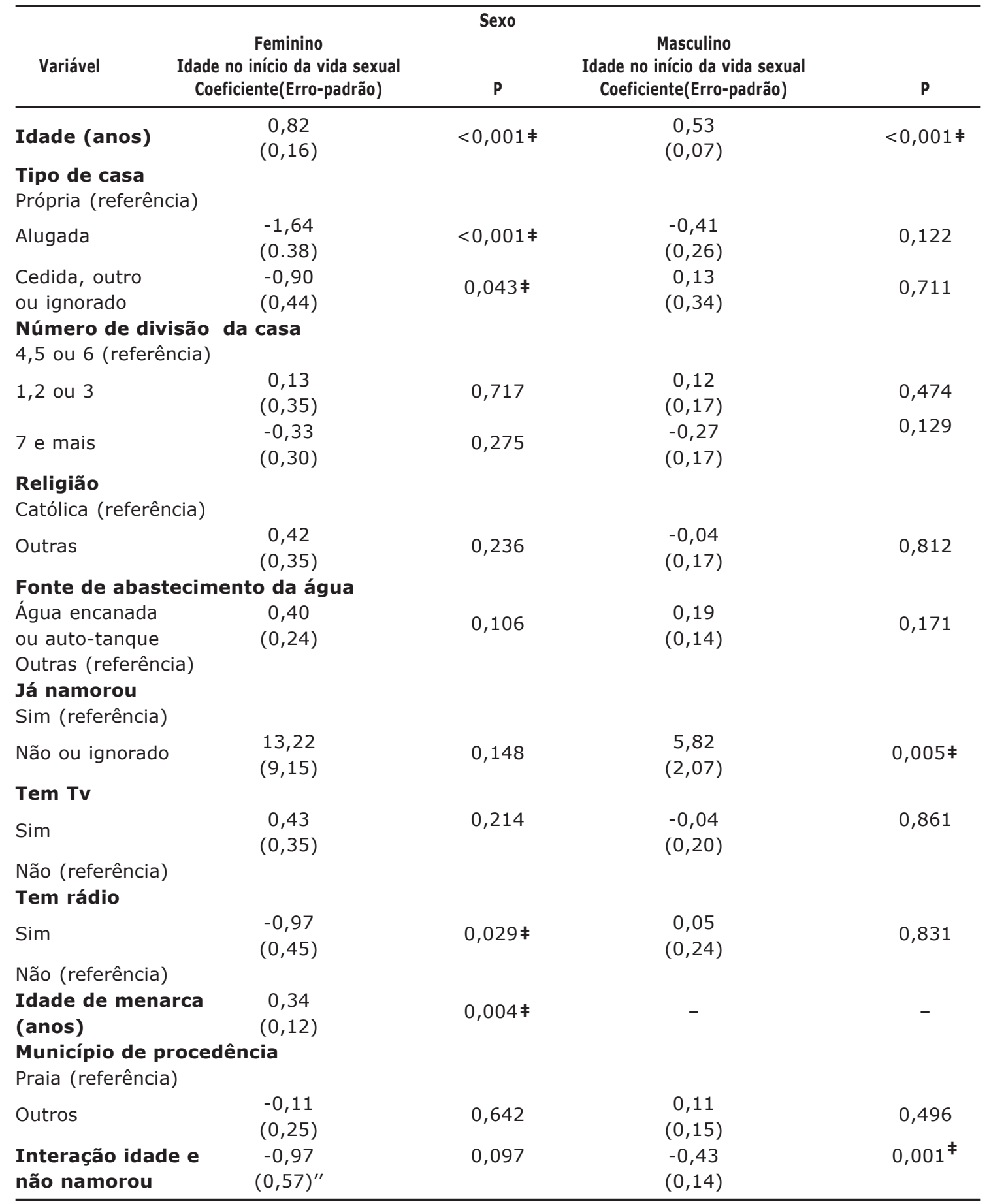

₹ $p<0,05=p$ descritivo pela regressão logística ordinal com múltipla co-variáveis foram apresentadas na tabela 2.

(-) significa que co-variáveis não foi importante para a explicação do evento de interesse.

A Tabela 2 apresenta as estimativas dos coeficientes e respectivos errospadrão e níveis descritivos dos fatores associados à idade do início da vida sexual para o modelo de regressão múltipla ordinal com função probit. 
Tabela 2: Estimativas do modelo reduzido de regressão múltipla ordinal com função probit para idade do início da vida sexual, segundo sexo, Ilha de Santiago, Cabo Verde, 2007

\begin{tabular}{|c|c|c|c|c|}
\hline $\begin{array}{r}\text { Idad } \\
\text { Co }\end{array}$ & $\begin{array}{l}\text { Feminino } \\
\text { início da vida sexual } \\
\text { iente(Erro-padrão) }\end{array}$ & Sexo & $\begin{array}{c}\text { Masculino } \\
\text { Idade no início da vida sexual } \\
\text { Coeficiente(Erro-padrão) }\end{array}$ & $\mathbf{P}$ \\
\hline Idade (anos) & $\begin{array}{c}0,72 \\
(0,14)\end{array}$ & $<0,001 \neq$ & $\begin{array}{c}0,51 \\
(0,06)\end{array}$ & $<0,001 \neq$ \\
\hline \multicolumn{5}{|c|}{$\begin{array}{l}\text { Tipo de casa } \\
\text { Própria, Cedida, outro ou NA } \\
\text { (referência) }\end{array}$} \\
\hline \multicolumn{4}{|l|}{$\begin{array}{l}\text { Já namorou } \\
\text { Sim (referência) }\end{array}$} & - \\
\hline Não ou ignorados & $\begin{array}{l}-1,70 \\
(0,63)\end{array}$ & $0,007 \neq$ & $\begin{array}{c}5,42 \\
(2,00)\end{array}$ & $0,007 \neq$ \\
\hline $\begin{array}{l}\text { Idade de menarca } \\
\text { (anos) }\end{array}$ & $\begin{array}{c}0,32 \\
(0,11)\end{array}$ & $0,004 \neq$ & - & - \\
\hline $\begin{array}{l}\text { Interação idade } \\
\text { e não namorou }\end{array}$ & - & - & $\begin{array}{l}-0,41 \\
(0,13)\end{array}$ & $0,002 \neq$ \\
\hline
\end{tabular}

As variáveis com $\mathrm{p}>0,05$ na Tabela 1 foram retiradas do modelo completo.

jovens mais novos só poderiam ter idade de início da vida sexual menor. Ainda, foi mais provável que seja significativamente menor a idade do início de vida sexual para meninas que moravam em casas alugadas e que não namoravam no momento da pesquisa. Além disso, quanto maior a idade da menarca, mais provável era que a idade de início da vida sexual fosse maior $(p=0,004)$. Para o sexo masculino, foi mais provável que seja significativamente maior a idade do início de vida sexual, quando o adolescente não namorava no período da entrevista.

\section{DISCUSSÃo}

A iniciação masculina ocorreu mais cedo do que a feminina, corroborando achados de estudos semelhantes ${ }^{12,13}$. Isso mostra que a iniciação sexual não tem ocorrido de forma similar entre homens e mulheres. Borges et al. sugerem que essa é uma tendência para grupos sociais ou entre gerações, indicando que diversos fatores complexos podem levar a tomada de decisão em iniciar a vida sexual ou adiar esse evento ${ }^{14}$. Por outro lado, estudos internacionais não têm considerado o adiamento do início da vida sexual como elemento pertinente para a política de prevenção de IST ou gravidez não planejada ${ }^{15,16}$. A iniciação sexual masculina faz parte de um ideário de constituição da identidade de gênero masculino, algo que se exprime na concretude dos comportamentos, o processo de constituição de pessoa, do "tornar-se homem" 17 .

A lógica das relações de gênero e a constituição de identidade de gênero em Cabo Verde é semelhante à observada no Brasil ${ }^{17,18}$. Assim, diferenças encontradas na iniciação entre sexos também são atribuídas à normatividade de gênero e à perspectiva de comportamentos distintos de homens e mulheres no campo da sexualidade ${ }^{18}$. A literatura sobre masculinidades afirma que tal processo também se faz por oposição e diferenciação a outros homens ${ }^{19}$.

Vários fatores têm sido mostrados como associados ao início da vida sexual, entre eles características individuais, tais como idade, cor, sexo, religião, escolaridade e a situação de trabalho. Além desses, são influenciadas por fatores familiares, como a comunicação e o relacionamento entre pais e filhos, a supervisão parental e a estrutura familiar ${ }^{14}$.

Quanto à idade de início da vida sexual, assim como outros estudos $7,20,0$ presente estudo aponta uma tendência crescente de redução dessa faixa etária, 
refletido em altas taxas de fecundidade entre adolescentes cabo-verdianas de 15 a 19 anos no início da década de $2000^{2}$.

Este estudo indica também que quanto maior a idade, mais provável que a idade do início da vida sexual seja maior e esse efeito é maior para meninas do que para os rapazes. A idade é uma variável que controla o efeito de que jovens mais novos só podem ter menor idade de início da vida sexual.

Na presente investigação, concluiuse que é mais provável que a idade do início da vida sexual para meninas fosse significativamente menor para aquelas que moravam em casas alugadas e que não namoravam no momento da pesquisa. Portanto, a condição de casa alugada é considerada uma Proxy para a condição econômica da família. Nesse sentido, as meninas com melhores condições econômicas iniciam a vida sexual mais tarde do que aquelas com piores condições. Contudo, não foram encontrados estudos que mostrassem essa relação entre morar em casas alugadas e a idade do início da vida sexual.

A menor frequência de início da vida sexual em adolescentes que não namoravam no momento da pesquisa em relação àquelas que namoravam corrobora achados de outros estudos em população adolescentes ${ }^{21}$.

Outro fator associado ao início da vida sexual em idades mais tardias foi a menarca também tardia, corroborando outros estudos ${ }^{19}$, o que pode ser explicado principalmente por questões biológicas que resultariam na maturação reprodutiva em idade mais tardia. Por outro lado, as meninas que têm a menarca em idade mais jovem (11 anos ou menos) não começam sistematicamente mais cedo sua vida sexual ${ }^{19}$. Exceto quando a menarca em idade mais jovem se atrela à escolarização curta (abaixo do nível fundamental completo) ${ }^{17}$.

No presente estudo, assim como em um estudo internacional consultado ${ }^{24-22}$, foi observada maior frequência de iniciação sexual protegida entre os jovens que se iniciaram sexualmente mais tarde.

A idade da iniciação sexual deve levada em consideração na formulação dos programas de prevenção à gravidez não planejada e ao IST/HIV, principalmente quando há práticas sexuais sem preservativo e com parceiros eventuais. Embora se saiba que cada sociedade possui um código, com normas relativas à idade apropriada ao exercício da sexualidade ${ }^{23}$, as relações sexuais sem preservativo e com parceiros eventuais constituem perigo independentemente da idade de iniciação sexual.

Os achados do presente estudo vão de encontro a dados do relatório do Ministério da Saúde de Cabo Verde ${ }^{24}$, no qual a vida sexual começa cada vez mais cedo em adolescentes e, na maior parte das vezes, sem informações adequadas. Os resultados do presente estudo mostram que, embora o início tenha sido cedo, os adolescentes se declararam informados sobre sexualidade. Na população estudada, havia um alto nível de informação sobre as formas mais conhecidas de contracepção - 93\% conheciam algum tipo de MAC antes da primeira relação sexual - e a maioria sabia que deveria usar preservativo. Isso pode ser efeito das ações do programa de saúde sexual e reprodutiva nas escolas secundárias ${ }^{21}$.

Consequentemente, a prevalência de HIV em Cabo Verde é bastante bai$\mathrm{xa}^{20}$, variando de $0,8 \%$ a $1,5 \%$ da população, contrariamente aos outros países da África Subsaariana, onde a epidemia parece estar estabilizada, com prevalência de $7,4 \%^{25}$. A porcentagem de HIV em adolescentes cabo-verdianos é aproximadamente $0 \%$.

Quanto às limitações do presente estudo, a taxa de resposta foi de $100 \%$, reduzindo assim a possibilidade de ocorrência de viés de seleção, tendo sido incluídos apenas adolescentes dos 13 aos 17 anos, com idade para o início da vida sexual. Todavia, as respostas podem ter sido influenciadas devido a vieses de declaração relacionados às expectativas de gênero ${ }^{15}$, isto é, os meninos podem ter relatado mais experiências sexuais e as meninas, menos. Deste modo, uma das limitações deste estudo é o possível viés de informação.

Outra limitação é o fato de não se ter medido algumas variáveis importan- 
tes no estudo da variável resposta em estudo. Por outro lado, no presente estudo mediram-se variáveis novas não encontradas em estudos semelhantes de inquérito sobre vida sexual de escolares.

A maior frequência de iniciação sexual protegida ocorre entre os jovens que se iniciaram sexualmente com ida-

\section{REFERÊNCIAS}

1. Warren CW, Santelli JS, Everett SA, Kann L, Collins JL, Cassell C, Morris L, Kolbe LJ. Sexual behavior among U.S. high school students, 19901995. Family Planning Perspectives, 30: 170-172, 200. 1998

2. Tavares CM, Camarano AA, Abreu LC. Fecundidade de mulheres Cabo-Verdianas - África Ocidental. Rev Bras Crescimento Desenvol Hum. 2008; 18(1): 1-10.

3. Rumo a um Novo Mundo: A vida sexual e reprodutiva de mulheres jovens; 1998. http://www.guttmacher. org/pubs/new_world_port.html (acessado em 29/Fev/2008.

4. World Health Organization. Adolescent Friendly Health Health Services - An agenda for Change. Geneva; 2002.

5. Diclemente RJ, Porton LE \& Hansen WB. New Directions for Adolescent Risk Prevention Research and Health Promotion Research and Interventions. Handbook of Adolescent Health Risk Behavior. Issues in Clinical Child Psychology. New York: Plenum Press. 1996.

6. Svare EI, Kjaer SK, Thomsen BL, Bock JE. Determinants for non-use of contraception at fi rst intercourse: a study of 10,841 young Danish women from the general population. Contraception. 2002;66(5):345-50.

7 Bozon M. A quel age lês femmes et lês hommes commencent-ils leur vie sexuelle? Comparasions et évolutions réecents. Pop et Soc 2003.

8. Borges ALV, Schor N. Início da vida sexual na adolescência e relações de gênero: um estudo transversal em São Paulo, Brasil, 2002. Cad Saúde Pública 2005; 21(2): 499-507. de mais avançada. Todavia, a influência de morar em casa própria ou cedida e de parceria afetivo-sexual no início da vida sexual revela a necessidade de ações de prevenção direcionadas para segmentos com piores condições sócioeconômicas e para adolescentes que não namoram.

9. Instituto Nacional de Estatística de Cabo Verde. Censo demográfico 2000. Praia: Instituto Nacional de Estatística de Cabo Verde; 2002

10. Castro MG, Abramovay M, Silva LB. Juventude e sexualidade. Brasília: UNESCO; 2004.

11. Greene, W. H. 2003 Econometric Analysis. 5th ed. Pearson Education.

12. Calazans G. Os jovens falam sobre sua sexualidade e saúde reprodutiva: elementos para refl exão. In: Abramo HW, Branco PM, organizadores. Retratos da juventude brasileira: análise de uma pesquisa nacional. São Paulo: Editora Fundação Perseu Abramo/ Instituto Cidadania; 2005. p.215-41.

13. Teixeira AMFB, Knauth DR, Fachel JMG, Leal AF. Adolescentes e uso de preservativos: as escolhas de jovens de três capitais brasileiras na iniciação e na última relação sexual. Cad Saude Publica. 2006;22(7):1385-96.

14. BORGES, Ana Luiza Vilela, LATORRE, Maria do Rosário Dias de Oliveira e SCHOR, Néia. Fatores associados ao início da vida sexual de adolescentes matriculados em uma unidade de saúde da família da zona leste do Município de São Paulo, Brasil. Cad. Saúde Pública, jul. 2007, vol.23, no.7, p.1583-1594.

15. Narring $F$, Wydler $H$, Michaud PA. First sexual intercourse and contraception: a cross-sectional survey on the sexuality of 16-20-year-olds in Switzerland. Schweiz Med Wochenschr. 2000;130(40):1389-98

16. Paiva V. Fazendo arte com camisinha: sexualidades jovens em tempos de AIDS. São Paulo: Summus; 2000.

17. Bozon M, Heilborn ML. Iniciação à sexualidade: modos de socialização, interações de gênero e trajetórias 
individuais. In: Heilborn ML, Aquino $E M L$, Bozon M, Knauth DR, organizadores. $O$ aprendizado da sexualidade. Rio de Janeiro: Garamond e Fiocruz; 2006. p. 156-205.

18. Varela Domingos A S. Programa de educação sexual em IST/HIV/SIDA com adolescentes de uma escola secundária de Cabo Verde: percepção dos atores envolvidos no programa. [Dissertação- Mestrado]. Bahia: Instituto de Saúde Coletiva, Universidade Federal; 2008. pp. 1-174.

19. Villela W V. homens que fazem sexo com mulheres. São Paulo: Nepaids, 1997.

20. Instituto Nacional de Estatística de Cabo Verde. Inquérito demográfico e de saúde reprodutiva 2005. Praia: Instituto Nacional de Estatística de Cabo Verde; 2007.

21. Tavares, Carlos Mendes; Schor, Néia; Franca Junior, Ivan and Diniz, Simone Grilo. Factors associated with sexual initiation and condom use among adolescents on Santiago Island, Cape Verde, West Africa. Cad. Saúde Pública [online]. 2009, vol.25, n.9, pp. 1969-1980 .

22. Paiva V, Calazans G, Venturi G, Dias R. Idade e uso de preservativo na iniciação sexual de adolescentes brasileiros. Rev. Saúde Pública 2008; 42 Suppl 1.

23. Heilborn M L. Entre as tramas da sexualidade de brasileira. Ver. Estud. Fem. 2006; 14(1):43-59.

24. BOZON, M. A quel age lês femmes et lês hommes commencent-ils leur vie sexuelle? Comparasions et évolutions réecents. Pop et Soc, 2003.

25. Rojas SHCC, Crestani KD, Batista GV, Melo APA. Características reprodutivas das adolescentes da legião mirim de Marília - São Paulo, Brasil, 2005. DST J Bras Doenças Sex Transm 2006; 18(2): 137-42. 